\title{
The Pathology of Lower Motor Neuron Disturbances in Relation to Treatment
}

\author{
By H. J. SEDDON, C.M.G., M.A., D.M., F.R.C.S. \\ Director of Studies, Institute of Orthopaedics, University of London, and Clinical Director of the Royal National \\ Orthopaedic Hospital.
}

W HEN a peripheral nerve has been severed the patient exhibits familiar and remarkably constant changes. Within the distribution of the nerve there is complete motor and sensory loss. Percutaneous stimulation of the nerve shows that its conductivity rapidly disappears; wasting of the affected muscles begins within a few days, fibrillation in these muscles begins on about the eighteenth day and the reaction of degeneration is fully established by the twenty-first. In the nerve itself Wallerian degeneration takes place, a breaking up and disappearance of the axons and their myelin sheaths. If the paralysis is extensive the limb is likely to become oedematous; this is due to the vasomotor paralysis, but still more to the paralysis of the muscles themselves which play an important part in the return of venous blood and lymph from the limb.

The muscles show two striking progressive changes, shrinkage of their fibres and interstitial fibrosis. But they are not in any peculiarly delicate state, such as that imagined by Keith, and do not require particularly gentle handling. Prolonged stretching is, however, harmful and it is chiefly for the prevention of it that splints or other forms of support are used.

In the absence of appropriate treatment contractures will develop and they are due to a number of factors:

1. The unopposed pull of normal muscles.

2. The shortening of the paralysed muscles, which is a consequence of the tendency to shrinkage of the fibrous tissue deposited in them.

3. Capsular shortening on the relaxed side of a joint and corresponding capsular lengthening on the stretched side.

4. A diffuse fibrosis involving many structuresjoints, muscles, tendon sheaths and subcutaneous tissueswhich is the result of the deposition of collagen fibres (fibrous tissue) in the stagnant oedema fluid of the swollen limb.

The first and second types of contracture cannot, of course, co-exist, and the second is usually due to inappropriate splinting, undertaken with the laudable intention of preventing the contracture of normal muscle but unaccompanied by regular passive movement of the affected part. It is still, alas, one of the commonest types of contracture and one of the most difficult to deal with.

Prevention of contractures is the first and most important duty of the physiotherapist, and there are three simple methods of treatment that must invariably be applied:

1. Elevation of the limb if the paralysis is at all extensive. In a complete lesion of the brachial plexus this may have to be maintained for several months; on the other hand in a simple ulnar paralysis there is rarely, if ever, need for it. It is designed to assist in the return flow of blood and lymph.

2. Passive movement of the affected part. This has three consequences: (a) It assists in the prevention of oedema by augmenting the return circulation.

(b) It maintains all muscles at their proper length.

(c) It prevents capsular contractures.

3. Splinting. Splints are frequently necessary to prevent stretching of the affected muscles and joint capsules and also to prevent shortening of normal muscles. But for the reason that $\mathbf{I}$ have already given the splinting must not take the form of rigid immobilization. Sometimes the splint must itself be rigid, but the possible evil consequence of this-namely, contracture of the relaxed paralysed muscles-can be prevented by passive movement of the limb out of the splint twice and, if necessary, more times a day. A better solution, where some power is retained in the affected part, is to give the patient what Capener has called a lively splint, an apparatus with a spring or elastic in it which allows the normal muscles a limited but highly beneficial range of activity.

The muscles themselves call for special attention in addition to the maintenance of their proper length. As was shown experimentally by Guttmann and others and clinically by Shirley Jackson, regular galvanic stimulation, at least daily, maintains muscle bulk to a remarkable degree and, it would appear, actually prevents interstitial fibrosis. There is no longer any doubt about the value of this treatment in conditions where re-innervation of the muscles may be expected.

After division of a nerve this re-innervation will occur only as a result of well-executed surgical repair, but a considerable period must elapse before re-innervation begins; the new axons travel distally from the site of suture at the rate of about one millimetre a day - an inch a month if you wish-so it may be nine or twelve months before any return of voluntary power can be expected. This recovery follows a fairly regular anatomical order, the order in which the motor branches are given off by the parent nerve trunk. So, as recovery progresses, the physiotherapist is required to continue the treatment appropriate for completely paralysed muscles in the distal distribution of the nerve while she is starting on the re-education of the proximal muscles that are beginning to show signs of life.

No matter how carefully a nerve has been repaired many of the outgrowing axons at the site of suture find their way into the wrong peripheral nerve tubes, and there is what we call an axonal confusion. A motor fibre may grow down a sensory tube, in which case it is completely useless. It may grow into a motor tube belonging to a muscle different from the one originally innervated by that particular axon. So we find that after, say, suture of the ulnar nerve the interossei have lost their delicate independent action, though they may act well enough together, as in the motion of grasping. This loss of independent movement is a great handicap, and the physiotherapist must do the best she can to restore a little independent movement by training the patient to contract the affected muscles individually. There is a strict biological limit to 
the success of such training, but it is none the less necessary to undertake it and at this stage occupational therapy that calls for fine individual movements of digits is especially valuable.

I now come to a second type of nerve damage which is very common but not perhaps quite so clearly recognized as the first, one that we have called axonotmesis, in which the axons of a nerve are completely interrupted but the tubes that contain them remain in continuity. If a nerve is squeezed very hard with smooth forceps the axons are interrupted just as completely as if the nerve had been cut across; a complete paralysis is produced with all the consequences that I have just described. Wallerian degeneration in the peripheral part of the nerve is indistinguishable from that following division of the nerve. But there is a striking difference at the site of injury. The tubes containing the nerve fibres are incredibly tough and will resist an immense amount of compression. Thus, when the compressing force has been removed the continuity of the nerve is maintained by these little tubes and they permit the axons to grow down them again along their old paths. Surgical repair is unnecessary because regeneration is spontaneous. Furthermore, there is no axonal confusion at the site of injury, and as the axons grow down they travel in their proper channels towards their appropriate destinations. Thus, although the immediate effects of the injury are indistinguishable from those following division of the nerve the ultimate recovery is very good indeed because there is a return of independent movement in the affected muscles and, sometimes equally important, of full sensibility in the previously denervated skin. There is no need, therefore, for the physiotherapist to bother so much about treatment designed to restore independent muscle action; it will return anyway. This is the sort of injury that commonly follows a fracture of the humerus involving the radial nerve.

There is a third and much more benign type of damage which we call neurapraxia. You are familiar with the comparatively transient paralysis that may follow the application of a tourniquet or the use of crutches that press too hard in the axilla. It is predominantly motor, there is little muscle wasting, the nerves retain their excitability below the level of injury and the muscles show no reaction of degeneration. The whole picture suggests a lesion in which the continuity of the axons is preserved, and we now know that the essential damage is loss of the myelin sheaths. These regenerate with comparative rapidity and the paralysis clears up within a matter of days or weeks. Furthermore, since there is no Wallerian degeneration and, therefore, no outgrowing of axons, there is no progressive march of recovery from the centre ot the periphery. Recovery usually occurs simultaneously throughout the distribution of the nerve. Here is a summary of the physiotherapeutic measures necessary in the three basic types of nerve injury, and it might be described as the $A B C$ of the treatment of lower motor neuron disorders.

\begin{tabular}{|c|c|c|c|}
\hline Treatment & $\begin{array}{l}\text { Nerve } \\
\text { Divided }\end{array}$ & Axonotmesis & Neurapraxio \\
\hline $\begin{array}{l}\text { Prevention of } \\
\text { oedema }\end{array}$ & Yes & Yes & No \\
\hline $\begin{array}{l}\text { Passive } \\
\text { movements }\end{array}$ & Yes & Yes & Yes \\
\hline Splinting & Yes & Yes & No \\
\hline Electro-therapy & Yes & Yes & No \\
\hline $\begin{array}{l}\text { Muscle } \\
\text { Re-education }\end{array}$ & Yes & No & No \\
\hline
\end{tabular}

I would like now to consider poliomyelitis, which is the commonest and most important of all spinal-cord disorders affecting the lower motor neuron. The essential damage is in the anterior horn cells, and it may range from a transient suspension of function to complete and irreparable destruction. It is well known that some parts of the cord are more vulnerable than others. We do not know all the reasons for this, though it appears that the virus itself has a particular affinity for anterior horn cells in certain regions. Here you see the distribution of lesions in cases seen in the Malta epidemic and it is fairly characteristic What is more, certain muscles are more vulnerable than others. In the lumbo-sacral region, which is the part most susceptible to damage, the tibialis anterior is the muscle most likely to suffer permanent paralysis. My colleague, Sharrard, has shown that this is due to the shortness of the column of anterior horn cells supplying it; other muscles with their anterior horn cells in the same part of the cord have longer cell stations. They are less likely to be completely knocked out by a localized focus of infection which we can liken in its effects to the explosion of a small bomb. A cottage will be completely blotted out; a whole terrace of houses will not. Of course, if the damage is overwhelming, all cell stations, long and short alike, are completely destroyed. Thus we find that if at the end of six to eight weeks-during which active contraction of the affected muscles has been attempted-there is still complete paralysis of all muscles in one segment of a limb, say below the knee, the prognosis is very bad: no worthwhile recovery can be expected. The peripheral consequences of the damage to the anterior horn cells take two forms. If all the anterior horn cells in, say, the lumbo-sacral region have been destroyed there is total paralysis of the lower limbs indistinguishable from that produced by section of all the nerve roots, except that sensibility is preserved. The motor axons degenerate, their conductivity is lost, the muscles fibrillate, they show the reaction of degeneration, they waste rapidly and become fibrotic. There is also a tendency to oedema, but not, perhaps, quite so great as after a nerve injury because there is no vasomotor paralysis. Contractures, too, are prone to develop, though if there are no surviving muscles there can be no deformity due to unopposed muscle action. On the other hand, where the anterior horn cells have been damaged but are still capable of recovery there is no axonal degeneration. Electrical excitability of the nerves is retained and is a remarkably accurate indication of the prospect of recovery. So far as we know there is no intermediate state in which axonal degeneration occurs and is followed by axonal regeneration, as after the repair of a divided nerve. The temporal pattern of recovery is much the same in all muscles, irrespective of their distance from the spinal cord. Furthermore, most of the recovery occurs fairly early. These two facts are powerful evidence against the occurrence of axonal regeneration, for if it were significant proximal muscles would recover sooner than the distal, and the whole process would be much slower than it actually. is. From the therapeutic point of view this is important. If a muscle is denervated it will remain so; and therefore maintenance of its volume by galvanic treatment is a waste of time. If a muscle, or part of it, is going to recover, its axonal connections are intact and therefore galvanism is unnecessary. This form of treatment has no place in the management of poliomyelitis.

From this rather streamlined account of the pathology of poliomyelitis you might conclude that recovery is solely dependent on the extent and intensity of the anterior horn cell damage, and that we can influence it but little. So far as the essential lesion is concerned I believe that this is the case, but there are, nevertheless, many useful and necessary things to be done. Poliomyelitis is the most ferocious of all deforming diseases. It is more crippling than rheumatoid arthritis or than joint tuberculosis; but fortunately we can do a great deal to prevent this evil consequence-indeed it is our first duty. The deformities produced by peripheral nerve injuries are trivial compared with those caused by poliomyelitis, simply because a nerve 
injury affects at most one limb whereas poliomyelitis can affect the whole body and produce a vicious and widespread upset of muscle balance. The measures that $I$ have described to you for the prevention of contractures after nerve injuries are strictly applicable in poliomyelitis and for the very same reasons.

1. The paralysed part needs to be elevated in the early days in order to prevent oedema, and the fibrosis and stiffness resulting from it. This applies chiefly to the upper limb: if the lower is affected the patient will be (or should be) recumbent for a number of weeks, and this suffices to prevent oedema. At this point we may conveniently remind ourselves that the only reason for using massage in the treatment of poliomyelitis is a poor peripheral circulation.

2. Paralysed and unaffected muscles alike must be maintained at their normal length by regular passive movement. Lengthening of paralysed muscles and stretching or shortening of joint capsules must be prevented where necessary by the application of splints, and here again the 'lively' splints devised by Capener are most valuable. In the adult simple support in bed is often all that is necessary; but a wriggling and unruly child requires splints if we are to avoid the contractures that come on with such astonishing rapidity in the early days of the disease and that are capable, at any rate in the child, of producing the most grotesque deformation.

Now we come to the much more debatable and, I am sure, unsettled question of muscle re-education. The difficulty is to know when to begin. There are three kinds of people treating poliomyelitis. There are the fixers, those who believe in putting on splints and keeping the patient very, very quiet. There are the floggers who get patients out of bed early and chase them round the physiotherapy department. . Then there are the good people, like myself, who are called festina-lentists who get the patients going very slowly. We do not really. know which of the three is right about this. To come to a conclusion, we require some knowledge. of what is happening in the spinal cord. How long is it before those cells that are going to recover are capable of transmitting the impulses set up by the patient's efforts to move the paralysed part? Is it possible to fatigue these convalescent cells by pushing them too hard? Ritchie Russell has shown that fatigue is an important factor in aggravating the severity and extent of cell damage during the stage of invasion. Is fatigue harmful during the phase of recovery? As I see it there are four ways of approaching this question:

1. It is possible to follow the actual histological changes in the anterior horn cells in post-mortem material obtained from patients dying at different periods after the onset of the disease. We are assured by Bodian that the whole cycle of changes in the cells that have survived is over five weeks after the onset, and so it might be assumed that treatment could safely be started at this period. On the other hand, Einarson has found appearances suggesting that anterior horn cells are still abnormal, yet perhaps capable of recovery, as late as eight months after the onset of the disease. What are we to do? My own feeling is that we ought not to drive purely histological evidence too far. Here, for example, are some brain cells from a patient who died of cerebral anoxia. She was completely unconscious and paralysed until her death three weeks later; yet although these cells had stopped working they look comparatively respectable. My own inclination is to err on the side of conservatism. I am encouraged in this attitude by the fact that the cerebro-spinal fluid sometimes remains abnormal for six weeks or.more after the onset of the disease, an indication that the inflammatory process in the spinal cord that follows the short sharp phase when the virus is active persists for some time. Furthermore, my colleague, Brooks, has shown that the electrical reactions of affected muscles do not settle down to a permanent state until about four months after the onset: during this period the condition of a proportion of the affected anterior horn cells is unstable.

Lastly, we have the evidence from treatment. I myself have found that early vigorous exercise of paretic muscles sometimes causes them to become weaker, and my experience is not unique. So until we know more about this awkward aspect of poliomyelitis it is wise to make haste slowly and to build up exercises gradually, lest by going too fast we overburden neuromuscular units that may barely have escaped dissolution.

\section{CHANGE OF ADDRESSES}

Mrs. M. Levy, the Journal Treasurer, has moved, to 105, Acacia Road, Blackheath, Johannesburg.

Mrs. D. Baumann has moved to 53/54, Colosseum Buildings, Market Square, East London.

Mrs. G. L. H. Diering has moved to 8, Pine Street, Plantation, Boksburg.

Miss D. Gibbon's address is c/o Standard Bank of S.A., Bloemfontein.

Mrs. J. Krogh's address is c/o Capt. J. Krogh, Military Camp, East London.

Mrs. S. Lewis (nee Robinson), is now at P.O. Box 37, Benoni.

Mrs. J. Medalie has moved to 15, Cyril Crescent, Cyrildene, Johannesburg.

Mrs. E. Metz has returned from Israel to 6, Delville Road, Germiston.

Capt. F. G. Ogg is now at P.O. Southbroom, South Coast, Natal.

Mr. R. Parker's address is c/o Mrs. Robinson, 87, Kritzinger Avenue, Brakpan.

Mrs. M. S. Robertson has moved to 455, 1st Avenue, Crown Deep, Crown Mines. 